\title{
Nasopharyngeal carcinoma: Advances in genomics and molecular genetics
}

\author{
ZENG ZhaoYang ${ }^{1,2}$, HUANG HongBin ${ }^{3,4}$, ZHANG WenLing ${ }^{3,4}$, XIANG Bo ${ }^{1,3}$, ZHOU Ming ${ }^{2,4}$, \\ ZHOU YanHong ${ }^{2,4}$, MA Jian ${ }^{1,3}$, YI Mei ${ }^{3,4,5}$, LI XiaYu ${ }^{3,4}$, LI XiaoLing ${ }^{3,4}$, XIONG Wei $^{3,4^{*}}$ \& LI \\ GuiYuan ${ }^{1,2,3,4^{*}}$ \\ ${ }^{1}$ Key Laboratory of Carcinogenesis and Cancer Invasion of Ministry of Education of China, Central South University, Changsha 410078, China \\ ${ }^{2}$ Key Laboratory of Carcinogenesis of Ministry of Health of China, Central South University, Changsha 410078, China \\ ${ }^{3}$ Disease Genome Research Center, Central South University, Changsha 410078, China \\ ${ }^{4}$ Cancer Research Institute, Central South University, Changsha 410078, China \\ ${ }^{5}$ Xiangya Hospital, Central South University, Changsha 410078, China
}

Received August 10, 2011; accepted September 1, 2011

Citation: Zeng Z Y, Huang H B, Zhang W L, et al. Nasopharyngeal carcinoma: Advances in genomics and molecular genetics. Sci China Life Sci, 2011, 54: 966-975, doi: 10.1007/s11427-011-4223-5

Nasopharyngeal carcinoma (NPC) is a squamous-cell carcinoma that arises in the epithelial lining of the nasopharynx [1]. This neoplasm has a notable ethnic and geographic distribution, being of high prevalence in southern China but rare in other parts of the world [2]. Familial clustering of NPC has been observed in diverse populations [3]. Elevated levels of circulating free Epstein-Barr virus (EBV) DNA and EBV-related antibodies in sera, as well as EBV DNA in tumor cells, have been consistently detected in individuals with NPC $[4,5]$. These studies have revealed that the risk factors of NPC are both environmental and genetic. How the risk factors interact, and the genes that are involved in the development of NPC, are not well understood [6].

\section{Genetic factors and GWAS analysis}

Early linkage analysis on Chinese sib-pairs with NPC suggested an association of particular human leukocyte antigen (HLA) haplotypes on chromosome 6p21 with susceptibility to NPC [7]. Supported by affected sib-pair haplotype sharing analysis and association studies on HLA regions, an

*Corresponding author (email: xiongwei@xysm.net; ligy@xysm.net)
NPC susceptibility locus was proposed, which was closely linked to the major histocompatibility complex but distinct from the HLA genes [8]. In addition, we and others have reported additional susceptibility loci on chromosomes 3 p21 [9,10], 4p15.1-q12 [11], and 5p13 [12] through linkage studies on NPC families from southern China. Many other candidate genes have also been implicated in NPC susceptibility [13-20].

Genome-wide association studies (GWAS) using state-of-the-art research tools are a rapid and cost-effective way to analyze the genetic differences between people with a specific illness and healthy individuals [21]. The purpose of these studies is to identify the genetic risk factors for the development or progression of disease. Recently, three groups have conducted GWAS for NPC in Taiwan, China [22], Malaysia [23], and Guangdong and Guangxi in Southern China [24], respectively. Consistent with previous reports, multiple independent associations within the HLA region, including $H L A-A, H L A-F, H L A-B / C$, and $H L A-$ $D Q / D R$, were revealed. The investigators hypothesized that some HLA antigens have reduced efficiency in activating the host immune response to EBV infection, which plays a critical role in the pathogenesis of NPC. These studies also suggested that certain genetic variations in GABBRI [22], 
ITGA9 [23], TNFRSF19, MDS1-EVI1, and CDKN2A [24] may influence susceptibility to NPC. Surprisingly, most of the single nucleotide polymorphisms associated with NPC are not in protein-coding regions of DNA. Instead, they are usually in the large intergenic regions, or in the introns that are edited out of the DNA sequence when mRNAs are processed. These are presumably motifs that control other genes or are in linkage disequilibrium with functional genomic regions, but in general, their function is not known.

\section{Transcriptomics}

Transcriptomics, also referred to as gene expression profiling, examines the variety, structure, function, and regulation of all transcripts in a given cell at a given time. It can illuminate the molecular mechanisms and regulatory networks involved in the various stages of NPC. Though suppression subtractive hybridization [25] has been successful in isolating tissue-specific genes in nasopharyngeal epithelial tissue [26,27] and candidate molecular markers of NPC [28], high-throughput techniques based on DNA microarrays are the most effective and convenient means to profile gene expression in NPC [29]. RNA-Seq is a recently developed approach to transcriptome profiling that uses deepsequencing technology; it is expected to provide useful information in NPC transcriptomics [30].

NPC tissue is heterogeneous and consists of cancer cells, numerous infiltrating inflammatory cells, and non-neoplastic nasopharyngeal epithelium and stroma. Therefore, obtaining specific groups of cells from tissue slides by microdissection is very important. Because the samples microdissected from NPC tissue are usually very small, RNA amplification is necessary for the subsequent experiments [31]. We [32-35] and others [36-38] have performed genome-wide transcriptome analysis by probing cDNA or oligonucleotide microarrays with fluorescently-labeled amplified RNA derived from laser-capture microdissected cells obtained from normal nasopharyngeal epithelium and areas of metaplasia-dysplasia and carcinoma from NPC. This approach enabled the identification of genes differentially expressed in each cell population, as well as numerous genes whose expression patterns helped to explain the aggressive clinical nature of this tumor type. For example, genes involved in the cell cycle [35] and those controlling invasive-metastatic potential were highly expressed in tumor cells. In contrast, genes under-expressed in these tumors included those involved in apoptosis, cell structure, and putative tumor suppressors [32]. The gene expression patterns also suggested alterations in the Wnt/beta-catenin and transforming growth factor beta pathways in NPC [33]. These results were further verified by a custom high-throughput tissue array platform [39,40]. Thus, expression profiling has indicated that the aberrant expression of growth, survival, and invasion-promoting genes may contribute to the molecular pathogenesis of NPC. One analysis method, known as Gene Set Enrichment Analysis, identifies co-regulated gene networks rather than individual genes that are up- or down-regulated in different cell populations [35,41]. This approach may help to identify useful markers of disease progression and novel potential therapeutic targets for NPC [42].

\section{Proteomics}

The transcriptome can be seen as a precursor for the proteome: that is, the entire set of proteins expressed by a transcriptome $[43,44]$. However, the levels of mRNA are not directly proportional to the levels of the proteins they encode. The number of protein molecules synthesized using a given template mRNA is highly dependent on the translation-initiation features of the mRNA sequence. There are two major strategies used for protein separation in proteomics: gel-dependent and gel-independent strategies [45]. In the gel-dependent strategy, two-dimensional gel electrophoresis (2-DE) coupled with mass spectrometry (MS) is commonly used to screen the "differential display" of proteins to compare their levels. In the gel-independent strategy, liquid chromatography (LC) or multi-dimensional LC replaces 2-DE to separate proteins or peptides, and these proteins or peptides are identified by MS $[46,47]$. LC-tandem MS (MS/MS) allows more definitive identification and quantitative determination of protein compounds [48,49]; this method complements the gel-dependent strategy. Surface-enhanced laser desorption/ionization time-of-flight MS (SELDI-TOF-MS) or SELDI ProteinChip technology [50] is a hybrid technology of retentate chromatography and matrix-assisted laser desorption/ionization (MALDI) TOF-MS, which allows protein capture, purification, and analysis on the ProteinChip surface and generates peaks in a mass spectrum (SELDI peaks) with unique mass-to-charge ratios detected by TOF-MS. Besides these "traditional" proteomics technologies, there has been much effort to develop new proteomics technologies to provide systematic proteomic data [51], including quantitative expression profiles [52-55], activity profiles, modification profiles, and interaction maps [56-61]. More advanced fragmentation methods in MS, such as electron capture dissociation and electron transfer dissociation [62], and chip-based proteomics strategies [63], including forward or reverse protein microarrays, have been used in proteomic modification profiles [64].

To understand NPC globally at the protein level, Li et al. [65] established a 2-DE reference map of NPC tissues and identified 216 landmark spots using MALDI-TOF-MS and electrospray ionization (ESI-Q)-TOF-MS. These authors constructed an NPC 2-DE/MS repository based on a Human Proteome Markup Language model with an open source XML database, Xindice.

An early diagnosis is one of the promises that proteomics 
hopes to fulfill. Recently, Cheng et al. analyzed the proteomes of NPC and normal nasopharyngeal epithelial tissues, and identified three potential biomarkers: stathmin, 14-3-3 sigma, and annexin I. Significant up-regulation of stathmin, and down-regulation of 14-3-3 sigma and annexin I, were observed in NPC and were significantly correlated with poor histologic differentiation, advanced clinical stage, recurrence, and poor prognosis $[66,67]$. Radiotherapy is the primary treatment for NPC, but radioresistance remains a serious obstacle to successful treatment in many cases. By comparing the protein profiles of radioresistant and radiosensitive cell lines, Feng et al. found that the down-regulation of 14-3-3 sigma and maspin and the up-regulation of GRP78 and Mn-SOD were significantly correlated with NPC radioresistance. Therefore, 14-3-3 sigma, maspin, GRP78, and Mn-SOD are potential biomarkers for predicting NPC response to radiotherapy [68]. Using proteomics, we analyzed the profile of serum peptides, also referred to as peptidome (the low molecular weight proteome) of NPC patients compared with normal control cases, and constructed diagnosis patterns for the early diagnosis of NPC [69].

Proteomics has also been used to explore the biological functions of novel NPC-associated genes in cellular signaling networks [70]. NGX6 [71,72], NAG7 [73,74], and BRD7 $[75,76]$ are novel genes cloned in our lab. To investigate their function, 2-DE was used to compare the protein expression profiles of NPC and control cell lines transfected with these genes. After staining and image analysis, differentially expressed protein spots were isolated and subjected to MS. We identified several proteins of interest that are regulated by these genes.

\section{Epigenetics}

Epigenetics is an important component of functional genomics. It can be defined as the study of the interplay between the environment and genetics, and the study of heritable changes that are not strictly dependent on the DNA sequence [77]. Epigenetic alterations, including DNA methylation [78,79], histone modification [80], chromatin remodeling [81,82], and non-coding RNA regulation [83], resulting in the loss of tumor suppressor gene function, are frequently involved in tumor development and progression [84]. Aberrant methylation of $\mathrm{CpG}$ islands on tumor suppressor genes such as NME1 [85], RASSFIA [86,87], UCHL1 [88], ANXA1 [89], and $S F N$ [90] is a characteristic epigenetic feature of NPC genomic DNA. MicroRNAs (miRNAs) constitute a family of small non-coding RNA molecules that are 22-25 nucleotides long [91-93]. Recent studies have demonstrated that miRNAs are intimately involved in the processes leading to NPC, such as gene regulation [94], cell growth [95], mitosis, tumor angiogenesis, invasion, and migration [96]. Exploring the relationship between miRNAs and the development of NPC will further the understanding of this cancer and provide new avenues for diagnosis and treatment [97].

\section{$5 \quad$ EBV and nanobacteria}

The human nasopharyngeal epithelium is directly exposed to all the toxic dusts and pathogens of the outside environment. Chronic inflammation of the nasopharynx caused by these pathogens is one important cause of NPC. EBV is an oncogenic human herpesvirus [98] that contains a DNA genome of $172 \mathrm{~kb}$. Latent membrane protein 1 (LMP1) is considered a viral oncogene [99], since it shows transforming activity in various cell types in vitro. Expression of LMP1 in immortalized nasopharyngeal epithelial cells induces an array of genes involved in growth stimulation, enhanced survival, and increased invasive potential [100-102]. The large EBV genome restricted previous studies to the study of a single oncogene. For the first time, we set up cell and animal models that allowed stable infection by the entire EBV genome [103]. This method provided the basis for whole-genome level investigations into the mechanism by which EBV contributes to NPC development [104]. We found that infection with the whole genome of EBV significantly promoted the growth and malignancy of epithelial cells [105] and activated NF-KB and MAPK signaling [106].

We first demonstrated the existence of nanobacteria (NB) by electron microscopy of a biopsy from an NPC patient, and then isolated NB for culture to verify our hypothesis [107]. NB are a new class of Gram-negative bacteria with sizes ranging from 50 to $400 \mathrm{~nm}$ diameter. We observed the co-existence of NB and EBV in NPC tissue, which resulted in the swelling of infected cells and inflammation in the relevant tissues. Electron micrographs of NPC tissue and in vitro culture studies showed that NB recruit calcium salts, and promote integration of the EBV genome into nasopharyngeal epithelial cells. EBV-infected cells then express oncogenes such as $L M P 1$ to promote their transformation into malignant tumor cells [108]. This is considered a direct early-stage pathway of NPC caused by NB and EBV. Besides promoting EBV infection, we showed that NB could secrete lipopolysaccharide, which stimulates the expression and release of cytokines such as TNF- $\alpha$, the expression of cytokine receptors such as CD14, TLR4 [109], and MD2, and the activation of p65, ERK1/2, and JNK1/2 that leads to $\mathrm{NF}-\mathrm{KB}$ and MAPK signaling [110]. Over-activation of these two pathways leads to cell proliferation and finally tumorigenesis. These data provided evidence that NB could induce NPC by mediating inflammation or the inflammation-cancer chain in the early phase of NPC. Taken together, we identified NB as a causative pathogen of NPC. From an etiological perspective, we showed that NB promoted infection by EBV and the inflammatory response of epithelial 
cells that induces NPC. The roles played by NB account for the major molecular events in the early phase of NPC.

\section{Important genes in NPC carcinogenesis}

SPLUNC1 is important in the early phase of NPC because it encodes a protein that inhibits $\mathrm{NB}$ and EBV activity. SPLUNC1 controls signaling through a gene-environmentmiRNA-target gene transduction pathway consisting of SPLUNC1-EBV/NB-miR141-PTEN, mediating the interaction between genes and the environment. The SPLUNC1 gene was isolated by suppression subtractive hybridization from a cDNA pool of adult nasopharyngeal epithelial cells and NPC cells [25,26], and it is uniquely expressed in nasopharyngeal epithelium [111]. Repeated gene-chip examination has demonstrated significant down-regulation of this gene in NPC [28,32,34]. We found that SPLUNC1 encodes a secreted protein that covers the surface of epithelial cells of the respiratory tracts. This protein could bind to the lipopolysaccharide of NB through its bactericidal/permeabilityincreasing domain to inhibit the growth of NB and other bacteria [107]. It could also inhibit tumorigenesis caused by EBV by promoting the lysis of EBV-infected cells, inhibiting the expression of oncogenes such as LMP1, promoting the expression of the gp350 viral protein that would enhance the immune response, and compromising the integrity of EBV [108]. These features suggest that SPLUNC1 is a part of the innate immune system [112], important in the defense against NB and EBV as well as being involved in the subsequent chronic inflammation they cause. In the case of EBV infection, the expression of LMP1 inhibited the expression of SPLUNC1 in nasopharyngeal epithelial cells, leading to loss of innate immunity. In addition, SPLUNCI may regulate miR141 [94] and the downstream PTEN-AKT signaling [113,114], and also the MAPK, NF- $\kappa \mathrm{B}$ [115], Bcl-2/Bax [116], and toll-like receptor [109,117-119] inflammatory response pathways [120], and ultimately the control of the cell cycle [121]. This pathway regulates the apoptotic cellular defense mechanism against cancer.

$B R D 7$ is a tumor suppressor transcription factor that regulates the cell cycle in the progressive phase of NPC. The transcription factor and target genes, MYC-BRD7-BRD2/ $B R D 3$, play important roles in NPC cell proliferation and the control of apoptosis. BRD7 was cloned by cDNA representational difference analysis combined with library screening [122,123]. BRD7 was found to inhibit the progress NPC cells from $G_{1}$ to $S$ phase, to induce apoptosis, slow cell growth, and reverse the malignancy of NPC cells [75]. Hybridization of a cell cycle-specific chip and a tumor-associated gene chip, and kinase activity analysis, showed that BRD7 could inhibit NPC development through regulation of the RAS/MEK/ERK, Rb/E2F [124,125], and WNT signaling pathways [126] during the progressive phase of NPC. BRD7 belongs to the bromodomain family and binds to acetylated histone $\mathrm{H} 3$, regulating the phosphorylation of the 10th lysine to transmit the transcription signal. BRD7 localizes in the nucleus, and contains a nuclear localization signal (amino acids 65-96) [127]. BRD2 and BRD3 belong to the same bromodomain family [128], and interact with $B R D 7$ to inhibit the cell cycle and trigger apoptosis. BRD7 enhances the transcription of BRD2 and $B R D 3$, suggesting that these two factors are downstream of $B R D 7$ in anti-NPC signaling. Upstream of $B R D 7$ signaling, the $5^{\prime}$ end of BRD7 ( -293 to $-186 \mathrm{bp}$ ) is a functional promoter containing binding sites for c-Myc and E2F6. c-Myc negatively regulates promoter activity and mRNA expression of BRD7 [129]. Moreover, the promoter region of $B R D 7$ is GC-rich and is regulated by methylation [130]; aberrant methylation is an important mechanism of $B R D 7$ dysfunction in NPC.

$N A G 7$ is another important regulator in the progressive phase of NPC, playing important roles in NPC cell proliferation and metastasis. NAG7 was cloned from chromosome 3 p25.3-26.3, the minimal common region of allelic loss among NPC patients [131]. NAG7 was found to be down-regulated in or absent from NPC biopsy tissues and cell lines. It functions by inhibiting cell cycle progression and promoting apoptosis [132]. We introduced gene chip [133] and proteomic [73] techniques when we studied this gene. We constructed differential gene and protein expression profiles induced by overexpression NAG7 in NPC cells, and found that our identified differentially expressed genes mainly participated in transcriptional regulation, cell growth, the cell cycle, cell metabolism, and apoptosis signaling transduction pathways. We also found that GASI was up-regulated at both the mRNA and protein levels upon $N A G 7$ overexpression, suggesting that NAG7 signaling might be mediated by GAS1. Our methodology combined the study of a single gene with both genomic and proteomic approaches, and we obtained the same results with both methods, providing an important basis for functional genomic studies of NPC. In further studies, we found that NAG7 could inhibit estrogen receptor alpha (ER- $\alpha$ ) [134] expression in NPC cells, activate the H-ras/p-c-raf, JNK/AP-1, and AKT/p70s6k signaling pathways, and regulate genes such as $C A V 1, M M P 1$ and $E Z R$, thus promoting the metastatic ability of NPC cells [135].

NOR1 encodes a mitochondria-reactive tumor suppressor protein (MiTS-NOR1) [136] with an antioxidant function, which was able to inhibit the Warburg effect in NPC cells to inhibit tumor growth $[137,138]$. Intracellular reactive oxygen species can cause damage to cellular proteins, DNA, and organelles, leading to genomic instability and cancer. Therefore antioxidation is an important cellular defense mechanism against cancer-inducing stimuli. MiTS-NOR1 is almost specifically expressed in the nasopharynx and tracheal epithelial cells [139], and was found to be regulated by the oxidative stress-related transcription factors heat shock transcription factor $1(H S F 1)$ and nuclear respiratory 
factor 1 (NRF1). We proved that oxidative stress, induced by treatment with hydrogen peroxide, could increase the expression of MiTS-NOR1 in normal nasopharyngeal epithelial cells through activation of HSF1 and NRF1, preventing further cellular damage [140]. Further studies with immunofluorescence, immuno-electron microscopy, and Western blotting confirmed that MiTS-NOR1 interacts with the ATP5O/OSCP subunit of mitochondrial ATP synthase to inhibit the expression of mitochondrial PDHK1 [141]. This inhibition would increase the efficiency of aerobic metabolism, increase the cellular ATP level, lower glucose consumption, and reduce the generation of lactic acid and glycolysis, all of which could reverse the Warburg effect in cancer cells. Additionally, MiTS-NOR1 could regulate the ratio of $\mathrm{Bax} / \mathrm{Bcl}-2$ in mitochondria, leading to the release of cytochrome $\mathrm{C}$, activating the mitochondrial apoptosis pathway through caspase 9 and 3, and therefore leading to the apoptosis of NPC cells.

The AP1-miR214-LTF regulatory axis is critical in NPC invasion and metastatic processes. Based on genetic linkage analysis of 18 human families at high-risk of NPC, we identified a 13.6-cM region on chromosome $3 \mathrm{p} 21$ that was tightly linked to the development of NPC $[9,10]$. We designed a gene chip containing all 288 genes in this region, and found that $L T F$ was significantly down-regulated in NPC tissues; this was further confirmed using a custom tissue chip containing 976 NPC tissue samples [142]. The down-regulation of $L T F$ was tightly associated with metastasis of NPC. Yi et al. [143] reported that heterozygous loss of the $L T F$ gene was found in $25 \%$ of NPC patients. Besides this, one or more point mutations, single nucleotide polymorphisms, or methylation of the promoter region of LTF could contribute to NPC susceptibility. The lactoferrin protein encoded by $L T F$ is widely distributed in mammalian tissues, milk, and other exocrine fluids such as tears, nasal secretions, and saliva, and it has antibacterial, antiviral, anti-inflammatory, and immune-regulatory functions as well as inhibition of tumor growth. We found that $L T F$ could inhibit NPC cell growth, arrest the cell cycle in stage $G_{0}-G_{1}$, down-regulate JNK2 and ERK in MAPK signaling, down-regulate cyclin D1 and phos-RB, and up-regulate p21 and p27. These findings suggest that LTF inhibits NPC growth by regulating both the cell cycle and the MAPK signaling pathway [142]. We also showed that LTF could inhibit the invasion and metastasis of NPC. An NPC cell line with re-expression of LTF showed reduced metastatic ability in vitro, and reduced tumorigenesis and lung metastasis in vivo when transplanted into nude mice [142]. The underlying mechanism is the inhibition of PDPK1 by LTF; PDPK1 controls the phosphorylation of AKT. LTF could also inhibit the membrane localization of $A K T$. PDPK1/AKT signaling plays important roles in tumor cell proliferation and metastasis. In a large-scale clinical investigation, we found that $L T F$ expression levels were negatively correlated with NPC metastasis, suggesting its potential as a prognostic marker [142]. We also demonstrated that LTF is a target gene of miR-214. The examination of normal nasopharyngeal tissue, nasopharyngeal primary tumor tissue, and NPC metastases showed that the expression level of $L T F$ was negatively correlated with that of miR-214, and miR-214 expression levels were significantly higher in metastatic NPC than in primary NPC [142]. The promoter region of miR-214 contains a binding site for the transcription factor AP-1, which is highly active in NPC tissue. AP-1 promoted the expression of miR-214 and inhibited $L T F$ expression, forming the AP1-miR214-LTF axis that underlies $L T F$ down-regulation in NPC tissues [142]. The inhibition of miR-214 expression significantly decreased the metastasis and invasion of NPC, suggesting a role for this axis in NPC development. Besides these findings, LTF inhibited the surface protein gp350 of EBV, and competed for the CD21 receptor on lymphocytes to inhibit the entry of EBV into the cells. $L T F$ also inhibited the activity of intracellular EBV. We further found that $L T F$ inhibited the migration of B cells, blocking B cell-mediated viral transmission from epithelial cells. The roles played by $L T F$ in preventing viral infection as well as in innate immunity are therefore critical in NPC development [142].

$N G X 6$ is another important protein in the anti-metastasis of NPC. NGX6 is a cellular surface glycoprotein containing an epidermal growth factor-like structural domain [144-150], which was found to be down-regulated in or absent from NPC biopsy tissues and cell lines [151-154]. $N G X 6$ has two transcripts of different sizes; NGX6a, the longer transcript, is involved in NPC development and is negatively correlated with lymph metastasis $[155,156]$. Restoring the expression of NGX6 significantly decreased the invasion and metastasis of NPC, improved the gap junction communication between cells, and reversed the malignancy of NPC cells [157]. Further studies showed that NGX6 regulated $K-R A S$ [158], $M E K 1$, and JUN expression, inhibited the phosphorylation of EGFR and MAPK kinase activity, up-regulated the expression of adhesion molecules such as $\mathrm{nm} 23$ and cadherin $\alpha 2$, and interacted with the cytoskeletal protein ezrin [151,159]. NGX6 inhibited the cell cycle and cell proliferation through the EGFR/ras/MEK/MAPK pathway $[144,160,161]$, and interacted with adhesion molecules to regulate the adhesion and migration of cells, altering the invasion and metastasis of the tumor [162].

Besides the above-mentioned genes, we and others have identified other genes involved in NPC carcinogenesis, including BMI1 [163,164], CCND1 [165], UBAP1 [166-169], STGC3 [170-173], NPCEDRG [174], RKIP [175], and $A B C B 1$ [176]. Some potential drugs [177], for example, an $\mathrm{N}$-sugar-substituted thalidomide analog [178] and 3,5-hydroxy-6,7,3',4'-tetramethoxyflavone [179] have been evaluated.

\section{Gene regulation networks in NPC}

We have reconstructed the activity spectrum of transcription 
factors and miRNAs in the different phases of NPC. We demonstrated dynamic changes in the activities of 26 transcription factors: for instance, the activities of AP2, ATF1, and ATF2 positively correlated with NPC severity [180,181]. Whereas the miRNAs miR34b/c, miR18a/b, and miR99a/b functioned as inhibitors of NPC, miR141 and miR200C were oncogenes for NPC [94]. Our combined analyses of the activity spectra and proteomics data showed that, although there was considerable inter-phase variability, the primary signaling pathways were MAPK, WNT, AKT, and NF- $\kappa$ B. These findings led to a "transcription factor $\rightarrow$ critical genes $\rightarrow$ miRNAs $\rightarrow$ target genes" regulatory network and a multistep model of NPC development, which comprises "environmental factors $\rightarrow$ SPLUC1 $\rightarrow$ BRD7/NAG7/ $N O R 1 \rightarrow$ LTF/NAX6 $\rightarrow$ tumor invasion and metastasis". We proposed the hypothesis that NPC is a disease of dysfunction in signal transduction and network regulation, and that the NPC susceptibility genes direct these multiple steps in sequence [182]. We believe that these susceptibility genes inform the progression of both the restrictive and susceptible checkpoints of NPC development, with one or more susceptibility genes being involved at each checkpoint. Several susceptible stages in this progression would constitute a chain in which all the susceptibility genes formed a highly interactive susceptibility gene group.

This work was supported by the National Natural Science Foundation of China (Grant Nos. 30871282, 30871365, 30971147, 81000883, 81000972, 81071644, 81071686, 81071756, 81171930, 81171931, 81171934, $81171988,81172189,81101509$, and 81102065), the 111 Project (Grant No. 111-2-12), the Natural Science Foundation of Hunan Province (Grant Nos. 10JJ7003, 11JJ1013, 11JJ22043 and 11JJ4072), and the Fundamental Research Funds for the Central Universities.

1 Lo K W, To K F, Huang D P. Focus on nasopharyngeal carcinoma. Cancer Cell, 2004, 5: 423-428

2 Wei W I, Sham J S T. Nasopharyngeal carcinoma. Lancet, 2005, 365: 2041-2054

3 Friborg J, Wohlfahrt J, Koch A, et al. Cancer susceptibility in nasopharyngeal carcinoma families-a population-based cohort study. Cancer Res, 2005, 65: 8567-8572

4 Chien Y C, Chen J Y, Liu M Y, et al. Serologic markers of Epstein-Barr virus infection and nasopharyngeal carcinoma in Taiwanese men. N Engl J Med, 2001, 345: 1877-1882

5 Lin J C, Wang W Y, Chen K Y, et al. Quantification of plasma Epstein-Barr virus DNA in patients with advanced nasopharyngeal carcinoma. N Engl J Med, 2004, 350: 2461-2470

6 Li G Y, Liu H Y, Zhou M, et al. The molecular mechanism involved in the development of nasopharyngeal carcinoma. Prog Biochem Biophys, 2006, 33: 922-931

7 Hildesheim A, Apple R J, Chen C J, et al. Association of HLA class I and II alleles and extended haplotypes with nasopharyngeal carcinoma in Taiwan. J Natl Cancer Inst, 2002, 94: 1780-1789

8 Lu S J, Day N E, Degos L, et al. Linkage of a nasopharyngeal carcinoma susceptibility locus to the HLA region. Nature, 1990, 346: 470-471

9 Xiong W, Zeng Z Y, Xia J H, et al. A susceptibility locus at chromosome 3 p21 linked to familial nasopharyngeal carcinoma. Cancer Res, 2004, 64: 1972-1974

10 Zeng Z Y, Zhou Y H, Zhang W L, et al. Family-based association analysis validates chromosome $3 \mathrm{p} 21$ as a putative nasopharyngeal carcinoma susceptibility locus. Genet Med, 2006, 8: 156-160

11 Feng B J, Huang W, Shugart Y Y, et al. Genome-wide scan for familial nasopharyngeal carcinoma reveals evidence of linkage to chromosome 4. Nat Genet, 2002, 31: 395-399

12 Hu L F, Qiu Q H, Fu S M, et al. A genome-wide scan suggests a susceptibility locus on 5p13 for nasopharyngeal carcinoma. Eur J Hum Genet, 2008, 16: 343-349

13 Zhou G Q, Zhai Y, Cui Y, et al. MDM2 promoter SNP309 is associated with risk of occurrence and advanced lymph node metastasis of nasopharyngeal carcinoma in Chinese population. Clin Cancer Res 2007, 13: 2627-2633

14 Xiong W, Zeng Z Y, Shen S R, et al. Studies of single nucleotide polymorphisms in UBAP1 gene and their association with nasopharyngeal carcinoma. Prog Biochem Biophys, 2002, 29: 766-770

15 Xiong W, Zeng Z Y, Xiao B Y, et al. Studies of association between nasopharyngeal carcinoma and single-nucleotide polymorphisms in NOR1, a novel oxidored-nitro domain-containing protein gene. Prog Biochem Biophys, 2003, 30: 401-405

$16 \mathrm{Yu} \mathrm{Y,} \mathrm{Zhu} \mathrm{S} \mathrm{G,} \mathrm{Xiang} \mathrm{J} \mathrm{J,} \mathrm{et} \mathrm{al.} \mathrm{Coding-region} \mathrm{single} \mathrm{nucleotide}$ polymorphisms in BRD7 gene and nasopharyngeal carcinoma susceptibility. Prog Biochem Biophys, 2001, 28: 568-572

17 Zeng Z Y, Xiong W, Shen S R, et al. High-throughput single nucleotide polymorphisms genotyping by dynamic allele-specific hybridization. Prog Biochem Biophys, 2002, 29: 806-810

18 Jia W H, Pan Q H, Qin H D, et al. A case-control and a family-based association study revealing an association between CYP2E1 polymorphisms and nasopharyngeal carcinoma risk in Cantonese. Carcinogenesis, 2009, 30: 2031-2036

19 He Y, Zhou G, Zhai Y, et al. Association of PLUNC gene polymorphisms with susceptibility to nasopharyngeal carcinoma in a Chinese population. J Med Genet, 2005, 42: 172-176

20 Xiong W, Zeng Z Y, Li X L, et al. Single-nucleotide polymorphisms in NGX6 gene and their correlation with nasopharyngeal carcinoma. Acta Biochim Et Biophys Sin, 2002, 34: 512-515

21 Sun Y L, Liu F, Zhao X H. Genome-wide association analysis based on copy number variations. Prog Biochem Biophys, 2009, 36: 968-977

22 Tse K P, Su W H, Chang K P, et al. Genome-wide association study reveals multiple nasopharyngeal carcinoma-associated loci within the HLA region at chromosome 6p21.3. Am J Hum Genet, 2009, 85: 194-203

$23 \mathrm{Ng} \mathrm{C} \mathrm{C,} \mathrm{Yew} \mathrm{P} \mathrm{Y,} \mathrm{Puah} \mathrm{S} \mathrm{M,} \mathrm{et} \mathrm{al.} \mathrm{A} \mathrm{genome-wide} \mathrm{association}$ study identifies ITGA9 conferring risk of nasopharyngeal carcinoma. J Hum Genet, 2009, 54: 392-397

24 Bei J X, Li Y, Jia W H, et al. A genome-wide association study of nasopharyngeal carcinoma identifies three new susceptibility loci. Nat Genet, 2010, 42: 599-U173

25 Zhang B C, Cao L, Qian J, et al. Construction of directional cDNA library from human embryo nasopharyngeal epithelia and screening a candidate tumor suppressor gene related with nasopharyngeal carcinoma. Prog Biochem Biophys, 2002, 29: 302-306

26 Zhang B C, Nie X M, Xiao B Y, et al. Identification of tissue-specific genes in nasopharyngeal epithelial tissue and differentially expressed genes in nasopharyngeal carcinoma by suppression subtractive hybridization and cDNA microarray. Gene Chromosomes Canc, 2003, 38: 80-90

27 Zhang B C, Zhou M, Zhou H D, et al. Characterization analysis of nasopharyngeal carcinoma differentially expressed gene PROL4. Prog Biochem Biophys, 2003, 30: 262-265

28 Zhou Y H, Zeng Z Y, Zhang W L, et al. Identification of candidate molecular markers of nasopharyngeal carcinoma by microarray analysis of subtracted cDNA libraries constructed by suppression subtractive hybridization. Eur J Cancer Prev, 2008, 17: 561-571

29 Huang $\mathrm{C}, \mathrm{Wu} \mathrm{M} \mathrm{H}, \mathrm{Li} \mathrm{G} \mathrm{Y}$. The present and advance in transcriptomics of nasopharyngeal carcinoma. Prog Biochem Biophys, 2007, 34: 1129-1135

30 Wang X, Wang X W, Wang L K, et al. A review on the processing and analysis of next-generation RNA-seq data. Prog Biochem Bio- 
phys, 2010, 37: 834-846

31 Zhou Y H, Zeng Z Y, Xiong W, et al. Microdissection and RNA line-amplification of nasopharyngeal carcinoma tissue. Prog Biochem Biophys, 2005, 32: 463-467

32 Zeng Z Y, Zhou Y H, Xiong W, et al. Analysis of gene expression identifies candidate molecular markers in nasopharyngeal carcinoma using microdissection and cDNA microarray. J Cancer Res Clin Oncol, 2007, 133: 71-81

33 Zeng Z Y, Zhou Y H, Zhang W L, et al. Gene expression profiling of nasopharyngeal carcinoma reveals the abnormally regulated Wnt signaling pathway. Hum Pathol, 2007, 38: 120-133

34 Zhou Y H, Zhang B C, Zeng Z Y, et al. Screening the differential expression genes of nasopharyngeal carcinoma using GenMAPP. Prog Biochem Biophys, 2005, 32: 1121-1129

35 Zhang W L, Zeng Z Y, Zhou Y H, et al. Identification of aberrant cell cycle regulation in Epstein-Barr virus-associated nasopharyngeal carcinoma by cDNA microarray and gene set enrichment analysis. Acta Biochim Et Biophys Sin, 2009, 41: 414-428

36 Sriuranpong V, Mutirangura A, Gillespie J W, et al. Global gene expression profile of nasopharyngeal carcinoma by laser capture microdissection and complementary DNA microarrays. Clin Cancer Res, 2004, 10: 4944-4958

37 Sengupta S, den Boon J A, Chen I H, et al. Genome-wide expression profiling reveals EBV-associated inhibition of MHC class I expression in nasopharyngeal carcinoma. Cancer Res, 2006, 66: 7999-8006

38 Wang S A, Li X, Li Z G, et al. Gene expression profile changes and possible molecular subtypes in differentiated-type nonkeratinizing nasopharyngeal carcinoma. Int J Cancer, 2011, 128: 753-762

39 Fan S Q, Xiao B Y, Cao L, et al. A new method to construct the cell microarrays and its application. Prog Biochem Biophys, 2004, 31: 756-760

40 Fan S Q, Zhang W L, Xiong W, et al. One novel tool and new methods to punch the graft tissues and make the receptive blocks for constructing the tissue microarrays. Prog Biochem Biophys, 2005, 32: 474-479

41 Hwang B, Li W T, Li W, et al. p-SAGE: Parametric statistical analysis of gene sets. Prog Biochem Biophys, 2009, 36: 1415-1422

42 Zhang W L, Zhou Y H, Xiao L, et al. Biomarkers of nasopharyngeal carcinoma. Prog Biochem Biophys, 2008, 35: 7-13

43 Ye H, Liu W, Yu C H, et al. Recent progress of proteomics in nonalcoholic fatty liver disease. Prog Biochem Biophys, 2011, 38: 487-498

44 Zhu Y M, Xu Q, Dong L R, et al. Identification of proteins associated with let-7a in gastric carcinoma cell line SGC-7901 by proteomics. Prog Biochem Biophys, 2011, 38: 441-448

45 Li F, Guan Y, Chen Z. Proteomics in nasopharyngeal carcinoma. Cell Mol Life Sci, 2008, 65: 1007-1012

46 Zhang C F, Tan T, Li J G, et al. Effect of fresh frozen plasma on endothelial cell migration diminishes during storage and its molecular mechanisms. Prog Biochem Biophys, 2011, 38: 528-535

47 Zhang R, Liu H H, Zhao H X, et al. Comparison of two protein extraction methods for proteomic analysis of chlorophyll-deficient mutants in Brassica juncea L. Prog Biochem Biophys, 2010, 37 : 1025-1032

48 Sun H C, Zhang J Y, Liu H, et al. Algorithm development of de novo peptide sequencing via tandem mass spectrometry. Prog Biochem Biophys, 2010, 37: 1278-1288

49 Zhang Z Q, Li M Y, Zhang G Y, et al. Quantitative proteome analysis of clinical gastric adenocarcinoma using O-18 stable isotype labeling and LCM. Prog Biochem Biophys, 2009, 36: 311-322

50 He Z G, Zhong H, Li Q H, et al. Research on the proteome response of Acidithiobacillus ferrooxidans to phosphate starvation by SELDI-Protein Chip technologies. Prog Biochem Biophys, 2008, 35 : 77-84

51 Chen L N, Wang Q, Shang Y K, et al. Human protein structural interaction network: Domain effects on network topology and protein function. Prog Biochem Biophys, 2010, 37: 517-526

52 Zhang W, Zhang J Y, Liu H, et al. Development of algorithms for mass spectrometry-based label-free quantitative proteomics. Prog Biochem Biophys, 2011, 38: 506-518

53 Li M X, Xiao Z Q, Peng F, et al. Quantitative proteomics analysis of $1 \mathrm{~cm}$ purified stroma of nasopharyngeal carcinoma and normal nasopharyngeal mucosa. Prog Biochem Biophys, 2009, 36: 1122-1133

54 Mi W, Wang J. Development of protein quantification based on ICP-MS. Prog Biochem Biophys, 2010, 37: 224-229

55 Liu Y F, Xiao Z Q, Zhang P F, et al. Quantitative proteomic analysis of metastasis-associated proteins in human primary lung adenocarcinoma. Prog Biochem Biophys, 2009, 36: 448-457

56 Ouyang Y M. The birth, development and applications of domain-domain interaction databases. Prog Biochem Biophys, 2009, 36: 280-287

57 Liu Z Y, Li D, Zhu Y P, et al. Progress in the evolutionary analysis of protein interaction networks. Prog Biochem Biophys, 2009, 36: $13-24$

58 Wei X L. Construction and analysis for molecular interaction network of cell adhesion. Prog Biochem Biophys, 2011, 38: 347-352

59 Zhao Y, Chen L N, Zhang L C, et al. Predicting disease genes of coronary artery disease based on functional consistency and network topological features. Prog Biochem Biophys, 2009, 36: 781-786

60 Sun H C, Li D, Wang J, et al. PNmerger: a cytoscape plugin to merge biological pathways and protein interaction networks. Prog Biochem Biophys, 2009, 36: 1613-1616

61 Li F, Bo X C, Li P, et al. Discovering active subnetwork in protein interaction network. Prog Biochem Biophys, 2010, 37: 208-217

62 Sun R X, Dong M Q, Chi H, et al. ECD/ETD-based tandem mass spectrometry in proteomics. Prog Biochem Biophys, 2010, 37: 94-102

63 Zhang J, Liu Q M, Xu D K, et al. In situ fabrication and application of protein microarray with cell-free system. Prog Biochem Biophys, 2009, 36: 391-397

64 Li N, Wu S F, Zhu Y P, et al. The progress of protein quality control methods in shotgun proteomics. Prog Biochem Biophys, 2009, 36: 668-675

65 Li F, Li M Y, Xiao Z Q, et al. Construction of a nasopharyngeal carcinoma 2D/MS repository with open source XML databaseXindice. BMC Bioinformatics, 2006, 7: 13

66 Cheng A L, Huang W G, Chen Z C, et al. Identification of novel nasopharyngeal carcinoma biomarkers by laser capture microdissection and proteomic analysis. Clin Cancer Res, 2008, 14: 435-445

67 Cheng A L, Huang W G, Zhang P F, et al. Comparative proteomics analysis of LCM purified nasopharyngeal carcinoma and normal nasopharyngeal epithelial tissue. Prog Biochem Biophys, 2008, 35: 521-530

68 Feng X P, Yi H, Li M Y, et al. Identification of biomarkers for predicting nasopharyngeal carcinoma response to radiotherapy by proteomics. Cancer Res, 2010, 70: 3450-3462

69 Zeng X, Zhao L, Li Z, et al. Impact of experimental and demographic variables in serum peptide profiling based on magnetic bead and MALDI-TOF mass spectrometry. Clin Chim Acta, 2010, 412: $112-119$

70 Liang K, Chen Z C, Yi H, et al. Screening of EGFR-regulated secreted proteins in human NPC cell line CNE2. Prog Biochem Biophys, 2007, 34: 100-106

71 Li J, Tan C, Xiang Q, et al. Search for differentially expressed proteins involved in the treatment of human nasopharyngeal carcinoma cells with NGX6 using two-dimensional electrophoresis and mass spectrometry. Prog Biochem Biophys, 2001, 28: 573-578

72 Li J, Tan C, Xiang Q, et al. Proteomic detection of changes in protein synthesis induced by NGX6 transfected in human nasopharyngeal carcinoma cells. J Protein Chem, 2001, 20: 265-271

73 Tan C, Li J, Wang J R, et al. Proteomic analysis of differential protein expression in human nasopharyngeal carcinoma cells induced by NAG7 transfection. Proteomics, 2002, 2: 306-312

74 Tan C, Li J, Xie Y, et al. Preliminary function study of NAG7 using two-dimensional electrophoresis and mass spectrometry. Acta Biochim Et Biophys Sin, 2001, 33: 373-378 
75 Peng C, Li X L, Zhou M, et al. Study of NPC-related gene BRD7 effect on NPC cell line CNE1. Prog Biochem Biophys, 2005, 32: 842-849

76 Peng C, Liang S P, Tan C, et al. Researching a novel NPC-related candidate suppressor gene BRD7 by two-dimensional gel electrophoresis and MALDI-TOF-MS. Acta Biochim Et Biophys Sin, 2003, 35: 816-822

77 Liu H Y, Peng S P, Zhou M, et al. Progress of epigenetic study on nasopharyngeal carcinoma. Prog Biochem Biophys, 2007, 34: 673-681

78 Feng W X, Wang K J, He B, et al. Method to analyze gene promoter methylation inhibition effect on binding of transcription factors. Prog Biochem Biophys, 2011, 38: 177-184

79 Fan S C, Zhang X G. Progress of bioinformatics study in DNA methylation. Prog Biochem Biophys, 2009, 36: 143-150

80 Jiang L H, Li Y X, Liu Q. Reconstruction of gene regulatory networks by integrating ChIP-chip, knock out and expression data. Prog Biochem Biophys, 2010, 37: 996-1005

81 Wang X L, Feng Y P, Zhao J, et al. The expression of p16 is regulated by the reversible histone acetylation. Prog Biochem Biophys, 2010, 37: 600-606

82 Jiang Z W, Liu X G, Zhou Z J. The regulation of histone modifications. Prog Biochem Biophys, 2009, 36: 1252-1259

83 Yan L M, Wu J Y, Yu X H, et al. Role of intronic microRNA in the regulation of endothelial nitric oxide synthase expression and the proliferation of endothelial cells. Prog Biochem Biophys, 2010, 37: $747-753$

84 Zhang $\mathrm{Z}$ P, Wu M H, Tang $\mathrm{H}$ L, et al. Effects of 5-aza-2'-deoxycitydine on proliferation of glioma cell lines and abnormal methylation of LRRC4 gene. Prog Biochem Biophys, 2009, 36: 904-909

85 Zhang W J, Yi B, Yi H, et al. Identification of hypermethylated genes in nasopharyngeal carcinoma cell line by proteomics. Prog Biochem Biophys, 2008, 35: 410-417

86 Lo K W, Kwong J, Hui A B, et al. High frequency of promoter hypermethylation of RASSF1A in nasopharyngeal carcinoma. Cancer Res, 2001, 61: 3877-3881

87 Yi M, Yang J, Chen X, et al. RASSF1A suppresses melanoma development by modulating apoptosis and cell-cycle progression. J Cell Physiol, 2011, 226: 2360-2369

88 Li L L, Tao Q, Jin H C, et al. The tumor suppressor UCHL1 forms a complex with p53/MDM2/ARF to promote p53 signaling and is frequently silenced in nasopharyngeal carcinoma. Clin Cancer Res, 2010, 16: 2949-2958

89 Tan S X, Hu R C, Dai A G, et al. DNA methylation inhibits ANXA1 gene expression in nasopharyngeal carcinoma cell lines. Prog Biochem Biophys, 2009, 36: 1319-1326

90 Tan $\mathrm{S} \mathrm{X}$, Li J, Yi $\mathrm{H}$, et al. Hypermethylation leads to down-regulated 14-3-3 sigma expression in nasopharyngeal carcinoma tissues. Prog Biochem Biophys, 2009, 36: 743-749

91 Jin Y F, Xu G M, Li Y, et al. A novel normalization approach for microRNA quantitative PCR. Prog Biochem Biophys, 2011, 38: 473-481

92 Guo Z Y, Mao C Q, Xiong L L, et al. The high throughput screening of direct regulatory microRNA and their target genes. Prog Biochem Biophys, 2009, 36: 1154-1164

93 Fu C, Lin K. Computational analysis of miRNA and target mRNA interactions: Combined effects of the quantity and quality of their binding sites. Prog Biochem Biophys, 2009, 36: 608-615

94 Zhang L M, Deng T, Li X Y, et al. microRNA-141 is involved in a nasopharyngeal carcinoma-related genes network. Carcinogenesis, 2010, 31: 559-566

95 Lu J A, He M L, Wang L, et al. MiR-26a inhibits cell growth and tumorigenesis of nasopharyngeal carcinoma through repression of EZH2. Cancer Res, 2011, 71: 225-233

96 Chen H C, Chen G H, Chen Y H, et al. microRNA deregulation and pathway alterations in nasopharyngeal carcinoma. Br J Cancer, 2009, 100: 1002-1011

97 Zhao L Q, Chen X, Cao Y. miRNA and nasopharyngeal carcinoma.
Chin Sci Bull, 2011, 56: 722-728

98 Wang X J. The molecular mechanism of herpesvirus membrane fusion. Prog Biochem Biophys, 2010, 37: 583-588

99 Fan S Q, Ma J, Zhou J, et al. Differential expression of Epstein-Barr virus-encoded RNA and several tumor-related genes in various types of nasopharyngeal epithelial lesions and nasopharyngeal carcinoma using tissue microarray analysis. Hum Pathol, 2006, 37: 593-605

100 Tang Y L, Lu J H, Cao L, et al. Genetic variations of EBV-LMP1 from nasopharyngeal carcinoma biopsies: potential loss of $\mathrm{T}$ cell epitopes. Braz J Med Biol Res, 2008, 41: 110-116

101 Lin X C, Liu S F, Luo X J, et al. EBV-encoded LMP1 regulates Op18/stathmin signaling pathway by cdc2 mediation in nasopharyngeal carcinoma cells. Int J Cancer, 2009, 124: 1020-1027

102 Zhang Z W, Zhang Q, Yu Y H, et al. Influence of Epstein-Barr virus encoded latent membrane protein 1-CTAR(3) for proteinic expression and cellular proliferation of NP69 cells. Prog Biochem Biophys, 2009, 36: 580-586

$103 \mathrm{Yu} \mathrm{Z} \mathrm{Y,} \mathrm{Lu} \mathrm{J} \mathrm{H,} \mathrm{Yu} \mathrm{H} \mathrm{B,} \mathrm{et} \mathrm{al.} \mathrm{A} \mathrm{precise} \mathrm{excision} \mathrm{of} \mathrm{the} \mathrm{complete}$ Epstein-Barr virus genome in a plasmid based on a bacterial artificial chromosome. J Virol Methods, 2011, doi:10.1016/j.jviromet. 2011.06.015

104 Zuo L L, Lu J H, Yu H B, et al. The observation of cell growth in the primary culture of transplantation tumor through GFP readout. Prog Biochem Biophys, 2011, 38: 563-567

105 Tang Y L, Lu J H, Wu M H, et al. Lytic replication and inductive production of recombinant Epstein Barr virus visualized. Prog Biochem Biophys, 2007, 34: 418-424

106 Lu J H, Tang Y L, Yu H B, et al. Epstein-Barr virus facilitates the malignant potential of immortalized epithelial cells: from latent genome to viral production and maintenance. Lab Invest, 2010, 90: 196-209

107 Zhou H D, Li G Y, Yang Y X, et al. Intracellular co-localization of SPLUNC1 protein with nanobacteria in nasopharyngeal carcinoma epithelia HNE1 cells depended on the bactericidal permeability increasing protein domain. Mol Immunol, 2006, 43: 1864-1871

108 Zhou H D, Li X L, Li G Y, et al. Effect of SPLUNC1 protein on the pseudomonas aeruginosa and Epstein-Barr virus. Mol Cell Biochem, 2008, 309: 191-197

109 Yang Y X, Yang Y B, Ming Z, et al. The study of nasopharygeal carcinoma cell 5-8F responsiveness to negative Gram's lipopolysaccharide via TLR4-regulating signaling pathway. Prog Biochem Biophys, 2007, 34: 138-145

110 Yang Y, Zhou H, Li W, et al. Lipopolysaccharide (LPS) regulates TLR4 signal transduction in nasopharynx epithelial cell line $5-8 \mathrm{~F}$ via NFkappaB and MAPKs signaling pathways. Mol Immunol, 2007, 44: 984-992

111 Zhou H D, Fan S Q, Zhao J, et al. Tissue distribution of the secretory protein, SPLUNC1, in the human fetus. Histochem Cell Biol, 2006, 125: 315-324

112 Zhou H D, Li X L, Li G Y. PLUNC family: Novel class of innate immune protective molecules in upper airway. Prog Biochem Biophys, 2004, 31: 767-771

113 Zhu M Y, Fu S G, Li M S, et al. Inhibited the activity of PTEN by alpha-fetoprotein caused resistance to all trans retinoic add of hepatoma cells. Prog Biochem Biophys, 2011, 38: 227-238

114 Matloob A, Pan L N, Pan H, et al. SMAD-4 cooperates with TGF-beta to enhance PTEN expression upon the inhibition of RAS/ERK pathway in gastric carcinoma cells. Prog Biochem Biophys, 2011, 38: 543-550

115 Cao D L, Yin K, Mo Z C, et al. Lipopolysaccharide down-regulates ABCA1 expression in foam cells in a nucleus factor-kappa B pathway-dependent manner. Prog Biochem Biophys, 2010, 37: 540-548

116 Zhang L, Xing D. Molecular mechanism of JNK/Bim/Bax apoptotic pathway induced by TNF-alpha in differentiated PC12 cells. Prog Biochem Biophys, 2010, 37: 370-380

117 Xue Y, Liu L L, He F, et al. IL-18 stimulates CSF-1 expression via MyD88-dependent pathway. Prog Biochem Biophys, 2011, 38: 311-319

118 Zhong T Y, Tang J, Chen D Y, et al. Using FRET to study the in- 
teraction domain of TLR4 binding to MD-2 in living cells. Prog Biochem Biophys, 2009, 36: 1451-1457

119 Sun B, Han D S. Negative regulation of toll-like receptors signaling pathways. Prog Biochem Biophys, 2009, 36: 1516-1522

120 Zhu X M, Yao Y M, Sheng Z Y. Inflammasome and Inflammatory Response. Inflammasome, 2010, 37: 129-137

121 Yang Y X, Yang Y B, Li X L, et al. The study of LPLUNC1 gene inhibits human nasopharyngeal carcinoma cell line HNE1 growth and proliferation. Inflammasome, 2007, 34: 366-374

$122 \mathrm{Yu}$ Y, Xie Y, Cao L, et al. Molecular cloning and functional primary study of a novel candidate tumor suppressor gene related with nasopharyngeal carcinoma. Prog Biochem Biophys, 2000, 27: 319-324

123 Nie X M, Zhang B C, Xiang J J, et al. Construction of prokaryotic expression vector of BRD7 and its expression in E. coli. Prog Biochem Biophys, 2002, 29: 631-634

124 Zhou J, Ma J, Zhang B C, et al. BRD7, a novel bromodomain gene, inhibits G1-S progression by transcriptionally regulating some important molecules involved in ras/MEK/ERK and $\mathrm{Rb} / \mathrm{E} 2 \mathrm{~F}$ pathways. J Cell Physiol, 2004, 200: 89-98

125 Li S F, Zhou M, Liu H Y, et al. The molecular mechanisms of $\mathrm{BRD} 7$ gene in regulating of Rb/E2F pathway. Prog Biochem Biophys, 2007, 34: 881-888

126 Peng C, Liu H Y, Zhou M, et al. BRD7 suppresses the growth of nasopharyngeal carcinoma cells (HNE1) through negatively regulating beta-catenin and ERK pathways. Mol Cell Biochem, 2007, 303: 141-149

127 Zhou M, Liu H, Xu X, et al. Identification of nuclear localization signal that governs nuclear import of BRD7 and its essential roles in inhibiting cell cycle progression. J Cell Biochem, 2006, 98: 920-930

128 Liu H Y, Li G Y. Advances in function analysis of a candidate tumor suppressor gene BRD7 and its family members. Prog Biochem Biophys, 2005, 32: 811-816

129 Liu H Y, Luo X M, Niu Z X, et al. Cloning and functional characterization of the regulation region of human BRD7 gene. Prog Biochem Biophys, 2006, 33: 531-539

130 Liu H Y, Zhang L M, Niu Z X, et al. Promoter methylation inhibits BRD7 expression in human nasopharyngeal carcinoma cells. BMC Cancer, 2008, 8: 253

131 Deng L, Jing N, Tan G, et al. A common region of allelic loss on chromosome region 3p25.3-26.3 in nasopharyngeal carcinoma. Gene Chromosomes Canc, 1998, 23: 21-25

132 Tan C, Li J, Wang J R, et al. Effect of NAG7 gene transfection on the growth of nasopharyngeal carcinoma cells. Prog Biochem Biophys, 2002, 29: 372-377

133 Tan C, Li J, Pen C, et al. The effects of NAG7 on gene expressional profile of HNE1 cells using cDNA microarray analysis. Prog Biochem Biophys, 2003, 30: 99-106

134 Gao Y, Chen X N, Wei C W, et al. c-abl upregulates the transcriptional activity of ER beta by their interaction. Prog Biochem Biophys, 2010, 37: 74-78

135 Huang C, Wu M, Tang Y, et al. NAG7 promotes human nasopharyngeal carcinoma invasion through inhibition of estrogen receptor alpha and up-regulation of JNK2/AP-1/MMP1 pathways. J Cell Physiol, 2009, 221: 394-401

136 Xu S J, Liu G L. Recent progress of mitochondrial dysfunction induced by beta-amyloid protein. Prog Biochem Biophys, 2010, 37: 589-593

137 Xiang B, Yi M, Wang L, et al. Preparation of polyclonal antibody specific for NOR1 and detection of its expression pattern in human tissues and nasopharyngeal carcinoma. Acta Biochim Et Biophys Sin, 2009, 41: 754-762

138 Nie X M, Gui R, Li D Q, et al. The effects of NOR1 on cells from human nasopharyngeal carcinoma cell line HNE1. Prog Biochem Biophys, 2005, 32: 777-780

139 Nie X M, Zhang B C, Li X L, et al. Cloning, expression, and mutation analysis of NOR1, a novel human gene down-regulated in HNE1 nasopharyngeal carcinoma cell line. J Cancer Res Clin Oncol, 2003, 129: 410-414

$140 \mathrm{Li} \mathrm{W}$, Li X, Wang W, et al. NOR1 is an HSF1- and NRF1-regulated putative tumor suppressor inactivated by promoter hypermethylation in nasopharyngeal carcinoma. Carcinogenesis, 2011, 32: 1305-1314

141 Xiang B, Wang L, Yi M, et al. Screen and identification of the protein-protein interactors of NOR1, a novel gene down-regulated in nasopharyngeal carcinoma. Prog Biochem Biophys, 2009, 36: 709-714

142 Zhou Y H, Zeng Z Y, Zhang W L, et al. Lactotransferrin: A candidate tumor suppressor-deficient expression in human nasopharyngeal carcinoma and inhibition of NPC cell proliferation by modulating the mitogen-activated protein kinase pathway. Int J Cancer, 2008, 123: 2065-2072

143 Yi H M, Li H, Peng D, et al. Genetic and epigenetic alterations of LTF at 3p21.3 in nasopharyngeal carcinoma. Oncol Res, 2006, 16: 261-272

144 Ma J, Zhou J, Fan S, et al. Role of a novel EGF-like domain-containing gene NGX6 in cell adhesion modulation in nasopharyngeal carcinoma cells. Carcinogenesis, 2005, 26: 281-291

145 Lian P, Guo Q, Peng Y, et al. The role of NGX6 gene on apoptosis of human colon cancer. Prog Biochem Biophys, 2008, 35: $1154-1160$

146 Wang X Y, Shen S R, Liu F, et al. Inhibitory effects of NGX6 gene on EGFR/K-ras/JNK/c-Jun/cyclin D1 signal pathway in the colon cancer. Prog Biochem Biophys, 2008, 35: 570-576

147 Guo Q, Shen S, Liao M, et al. NGX6 inhibits cell invasion and adhesion through suppression of Wnt/beta-catenin signal pathway in colon cancer. Acta Biochim Biophys Sin (Shanghai), 2010, 42: 450-456

148 Liu M, Peng Y, Wang X, et al. NGX6 gene mediated by promoter methylation as a potential molecular marker in colorectal cancer. BMC Cancer, 2010, 10: 160

149 Peng Y, Li H, Wu M, et al. NGX6 inhibits AP-1 and Ets-1 expression and down-regulates cyclin D1 in human colorectal cancer. Acta Biochim Biophys Sin (Shanghai), 2009, 41: 504-514

150 Guo Q, Wu M, Lian P, et al. Synergistic effect of indomethacin and NGX6 on proliferation and invasion by human colorectal cancer cells through modulation of the Wnt/beta-catenin signaling pathway. Mol Cell Biochem, 2009, 330: 71-81

151 Ma J, Li J, Zhou J, et al. Profiling genes differentially expressed in NGX6 overexpressed nasopharyngeal carcinoma cells by cDNA array. J Cancer Res Clin Oncol, 2002, 128: 683-690

152 Zhang X M, Wang X Y, Sheng S R, et al. Expression of tumor related genes NGX6, NAG-7, BRD7 in gastric and colorectal cancer. World J Gastroenterol, 2003, 9: 1729-1733

153 Fan S Q, Zhang W L, Xu L N, et al. Investigation of clinical significance and expression of tumor metastasis-related genes in the nasopharyngeal cancer using tissue microarray technique. Prog Biochem Biophys, 2009, 36: 616-623

154 Fan S Q, Zhang W L, Zhou M, et al. Study of in situ expression of NGX6 gene in the several common types of cancer and its clinical significance. Prog Biochem Biophys, 2008, 35: 1014-1020

155 Wang L, Xiang B, Yi M, et al. Identification of a new seven-span transmembrane protein: NGX6a is downregulated in nasopharyngeal carcinoma and is associated with tumor metastasis. J Histochem Cytochem, 2010, 58: 41-51

156 Wang L L, Zhang Q H, Ma J, et al. Role of NGX6 in nasopharyngeal carcinoma with high ability of metastasis cell line 5-8F. Prog Biochem Biophys, 2005, 32: 618-624

157 Liu M J, Wang X Y, Shen S R, et al. Cloning and identification of promoter of suppressed-tumor gene NGX6. Prog Biochem Biophys, 2010, 37: 1082-1089

158 Zhang H D, Wang X N, Zhou Z, et al. Mutation detection of K-ras gene in paraffin-embedded colorectal cancer tissues by using chip-based TGCE. Prog Biochem Biophys, 2010, 37: 794-800

159 Peng S, Fan S, Li X, et al. The expression of ezrin in NPC and its interaction with NGX6, a novel candidate suppressor. Cancer Sci, 2007, 98: 341-349

160 Peng S P, Li X L, Wang L, et al. The role of NGX6 and its deletion mutants in the proliferation, adhesion and migration of nasopharyngeal carcinoma 5-8F cells. Oncology, 2006, 71: 273-281 
161 Wang L L, Ma J, Li J, et al. NGX6 gene inhibits cell proliferation and plays a negative role in EGFR pathway in nasopharyngeal carcinoma cells. J Cell Biochem, 2005, 95: 64-73

162 Wang X Y, Wu M H, Liu F, et al. Differential miRNA expression and their target genes between NGX6-positive and negative colon cancer cells. Mol Cell Biochem, 2011, 345: 283-290

163 Song L B, Zeng M S, Liao W T, et al. Bmi-1 is a novel molecular marker of nasopharyngeal carcinoma progression and immortalizes primary human nasopharyngeal epithelial cells. Cancer Res, 2006, 66: 6225-6232

164 Song L B, Li J, Liao W T, et al. The polycomb group protein Bmi-1 represses the tumor suppressor PTEN and induces epithelial-mesenchymal transition in human nasopharyngeal epithelial cells. J Clin Invest, 2009, 119: 3626-3636

165 Hui A B Y, Or Y Y Y, Takano H, et al. Array-based comparative genomic hybridization analysis identified cyclin D1 as a target oncogene at 11q13.3 in nasopharyngeal carcinoma. Cancer Res, 2005, 65: 8125-8133

166 Xiao B, Fan S, Zeng Z, et al. Purification of novel UBAP1 protein and its decreased expression on nasopharyngeal carcinoma tissue microarray. Protein Expr Purif, 2006, 47: 60-67

167 Qian J, Dong L, Zhang B C, et al. Identification and digitalized expression analysis of murine UBAP1 gene by means of EST database searching. Prog Biochem Biophys, 2002, 29: 323-327

168 Zeng Z Y, Xiong W, Zhou Y H, et al. Effect of UBAP1 gene transfection on the growth of nasopharyngeal carcinoma cells. Prog Biochem Biophys, 2005, 32: 929-936

169 Qian J, Yang J, Zhang X, et al. Isolation and characterization of a novel cDNA, UBAP1, derived from the tumor suppressor locus in human chromosome 9p21-22. J Cancer Res Clin Oncol, 2001, 127: 613-618

170 Li L, He X S, Luo Q A, et al. Effect of STGC3 gene deletion mutant on the growth of CNE2 cells. Prog Biochem Biophys, 2011, 38: 248-253

171 Qiu Q C, Bo H, He X S, et al. Tumorigenicity investigation of CNE2 cell line STGC3 expression induced by tet-on system in nude mice. Prog Biochem Biophys, 2007, 34: 359-365

172 Hu B, Qiu Q C, He X S, et al. Effect of estrogen on CNE2 cell line transfected with STGC3 gene. Prog Biochem Biophys, 2007, 34: 538-545

173 Deng M, He X S, Luo Q, et al. Tet regulating express on system establishment and functional analysis of novel gene STGC3 in nasopharyngeal carcinoma cell line CNE2. Prog Biochem Biophys, 2006, 33: 39-44

174 Yang S, Hu H, Deng M, et al. Effect of the novel gene NPCEDRG associated with NPC on the growth of CNE2 cells. Prog Biochem Biophys, 2010, 37: 167-174

175 Yan C, Jun L, Hong Y, et al. RKIP downregulation associated with the metastasis and activation of NF-kappa B signaling pathway in nasopharyngeal carcinoma. Prog Biochem Biophys, 2009, 36: 880-889

176 Xie S M, Du S S, Fang W Y, et al. Expression of ten ABC transporters in cisplatin-resistant nasopharyngeal carcinoma cell lines. Prog Biochem Biophys, 2009, 36: 458-463

177 Liu W, Xie H W. Potential drug target discovery based on bioinformatics methods. Prog Biochem Biophys, 2011, 38: 11-19

178 Yi W Y, Xu B, Li M, et al. Reversal effect of a novel N-sugar substituted thalidomide analogue on multidrug resistant human nasopharyngeal carcinoma cells. Prog Biochem Biophys, 2009, 36: 58-64

179 Cao C S, Shen W Z, Li Y L, et al. 3,5-Hydroxy-6,7,3',4'-tetramethoxyflavone isolated from Laggera pterodonta induces CNE cell apoptosis. Prog Biochem Biophys, 2011, 38: 254-261

180 Su B, Tang H L, Deng M, et al. Stage-associated dynamic activity profile of transcription factors in nasopharyngeal carcinoma progression based on protein/DNA array analysis. OMICS, 2011, 15: $49-60$

181 Su B, Xiang B, Wang L, et al. Profiling and comparing transcription factors activated in non-metastatic and metastatic nasopharyngeal carcinoma cells. J Cell Biochem, 2010, 109: 173-183

182 Wu M, Li X, Li G. Signaling transduction network mediated by tumor suppressor/susceptibility genes in NPC. Curr Genomics, 2009, 10: $216-222$

Open Access This article is distributed under the terms of the Creative Commons Attribution License which permits any use, distribution, and reproduction in any medium, provided the original author(s) and source are credited. 